\title{
Factors affecting treatment, management and mortality in cases of retroperitoneal hematoma after cardiac catheterization: a single-center experience
}

\author{
Ebubekir Gündeş ${ }^{1}$, Ulaş Aday ${ }^{1}$, Mustafa Bulut ${ }^{2}$, Hüseyin Çiyiltepe ${ }^{1}$, Durmuş Ali Çetin ${ }^{1}$, Selçuk Gülmez ${ }^{1}$, \\ Aziz Serkan Senger ${ }^{1}$, Kamuran Cumhur Değer ${ }^{1}$, Erdal Polat ${ }^{1}$, Mustafa Duman ${ }^{1}$ \\ ${ }^{1}$ Gastroenterological Surgery Department, Kartal Koşuyolu High Speciality and Training Hospital, Istanbul, Turkey \\ ${ }^{2}$ Cardiology Department, Kartal Koşuyolu High Speciality and Training Hospital, Istanbul, Turkey
}

Adv Interv Cardiol 2017; 13, 3 (49): 218-224

DOI: https://doi.org/10.5114/aic.2017.70189

\begin{abstract}
Introduction: There is little information available on the clinical aspects, results, treatment and management of cardiac catheterization-related retroperitoneal hematoma. Data on the subject are rather limited, and current publications are based on a limited number of retrospective cohort studies and case reports.

Aim: To perform a retrospective analysis of the demographic, clinical, and in-hospital results of patients who developed retroperitoneal hematoma (RPH) after cardiac catheterization (CC).

Material and methods: The cases of 124,064 patients who had CCs between January 2010 and October 2016 were retrospectively analyzed. Patients diagnosed with RPH were classified into three groups depending on the method of treatment: conservative (group 1), endovascular stenting (group 2), and surgery (group 3). The independent risk factors, based on RPH-related mortality, were determined by logistic regression analysis.

Results: Of the 68 (0.054\%) patients with RPH, 75\% received conservative treatment, $13.2 \%$ underwent angiographic stent placement, and $11.7 \%$ had surgical treatment. Red blood cell packets (RBCPs) $(p=0.043)$, duration of hospitalization $(p=0.007)$, and mortality rates $(p=0.006)$ were statistically significantly higher in group 3 in comparison to the other groups. Multivariate subgroup analysis was conducted to determine mortality rates, with post-procedural highest creatinine $\geq 2 \mathrm{mg} / \mathrm{dl}$ and RBCPs $\geq 10$ established as independent risk factors.

Conclusions: Hemodynamically stable patients with no active hemorrhaging are shown to have good results with conservative treatment. We believe that endovascular methods should be used initially for hemodynamically unstable patients, while surgical treatment should be employed in cases where endovascular methods fail or abdominal compartment syndrome develops.
\end{abstract}

Key words: cardiac catheterization, retroperitoneal hematoma, abdominal surgery.

\section{Introduction}

Retroperitoneal hematoma $(\mathrm{RPH})$ most frequently occurs as a complication of femoral arterial catheterization and pelvic or lumbar trauma. The number of serious extra-cardiac complications has been increasing in relation to the number of complex percutaneous coronary interventions (PCls) performed. Major hemorrhaging complications, which are among the most common post-PCl complications, are associated with prolonged hospitalization, morbidity, and mortality risks [1, 2]. Although some studies have reported that the incidence rate of post-PCI RPH varied from $0.4 \%$ to $0.74 \%$, the actual incidence rate could be higher because of unreported or undetected cases [3-5].
Contrary to other hemorrhaging areas, the retroperitoneal space can contain a large volume of blood until hypovolemic symptoms appear, an event that could lead to delayed diagnosis, morbidity, and mortal results [5]. Although there have been a number of studies on post-cardiac catheterization (post-CC) complications, the studies on RPH have been limited. As a result, a suitable treatment for RPH patients has not yet been well defined.

\section{Aim}

The aim of this study was to apply a case-controlled study design in an analysis of the clinical reflections of

\section{Corresponding author:}

Ebubekir Gündeş MD, Gastroenterological Surgery Department, Kartal Koşuyolu High Speciality and Training Hospital, Denizer cad. No: 22, 34000 Istanbul, Turkey, phone: +90 5058606740, e-mail: ebubekir82@hotmail.com

Received: 3.03.2017, accepted: 19.04.2017. 
$\mathrm{RPH}$, treatment procedure determinants, and factors affecting mortality at a single center.

\section{Material and methods}

\section{Study design}

This retrospective observational study was conducted at Kartal Koşuyolu High Speciality and Training Hospital, a tertiary-level training and research hospital. Informed consent documents were obtained from all the patients covered by the study for pre-procedural diagnosis and interventional coronary catheterization. Following the approval of Kartal Koşuyolu High Speciality and Training Hospital's Board of Clinical Research Evaluation (Registration No; 2016.5/7-18), patients with CC-related RPH were included in the study.

\section{Study population and categorization}

Inclusion criteria: cases with RPH following CCS that were conducted at our center between January 2010 and October 2016.

Exclusion criteria: cases with RPH unrelated to CC and patients with incomplete records.

The patients were divided into three groups depending on the method of treatment. Patients who received conservative treatment were assigned to group $1(n=51)$, those who had angiographic treatment were assigned to group $2(n=9)$, and those who underwent surgical treatment were assigned to group $3(n=8)$. The diagnostic procedure scheme is shown in Figure 1. The subgroup analysis was conducted in two groups: those with mortality $(n=14)$ and those without $(n=54)$.

\section{Vascular closure devices}

A Bioabsorbable Polyglycolic Plug (EXOSEAL, Cordis Corporation, Bridgewater, NJ, USA) or a sutured (Perclose ProGlide; Abbott Vascular, Redwood Shores, Calif.) vascular closure device (VCD) was used to repair the femoral artery puncture defect depending on the operator preference in selected patients.

\section{Data}

Data for the study were collected using standardized definitions and a non-standardized data collection form.
Data on the patients' pre-CC age, sex, comorbidity, CC indication, weight, body surface area, body mass index (BMI), left ventricular ejection fraction (LVEF), laboratory analysis results (hematocrit (hct), creatinine (cre), platelet (plt)), and antiaggregant and/or anticoagulant treatments were recorded.

The post-CC time of RPH diagnosis, laboratory analysis results (minimum hct and plt levels, maximum cre level, and the amount of hct decrease), the maximum size and localization of the hematoma as shown by abdominal computed tomography (CT), the need for blood transfusion, duration of hospitalization, and mortality cases were also recorded.

All the data were recorded in Excel tables without any identifying information in order to maintain the anonymity of the patients.

\section{Statistical analysis}

Statistical Package for the Social Sciences (SPSS 22 Inc., Chicago, IL, USA) software was used for the biostatistical analyses. The data of the patients included in the study were reported in mean figures, standard deviation values, and percentages where necessary. The distribution of data was assessed by the Kolmogorov-Smirnov test. The ANOVA test was used in multi-group comparisons of normally distributed data. The multi-group comparisons of non-parametric data were evaluated using the Kruskal-Wallis analysis. Categorical groups were compared by the $\chi^{2}$ test; $p<0.05$ was set as the statistical significance level. At the development stage of the multivariate logistic regression model, a univariate logistic regression analysis of each variable was conducted to ascertain the candidate variables for RPH-related mortality factors. In cases where the probability value of the Wald test statistic was smaller than the level of significance $(p<0.25)$, related variables were included in the multivariate model. The odds ratio (OR) and 95\% confidence interval $(95 \% \mathrm{Cl})$ were calculated, while statistical significance was set at $p<0.05$.

\section{Results}

Of the 124,064 patients who had CCs during the years covered in the study, 68 (0.054\%) contracted RPH. An evaluation of all the cases revealed that patients with no

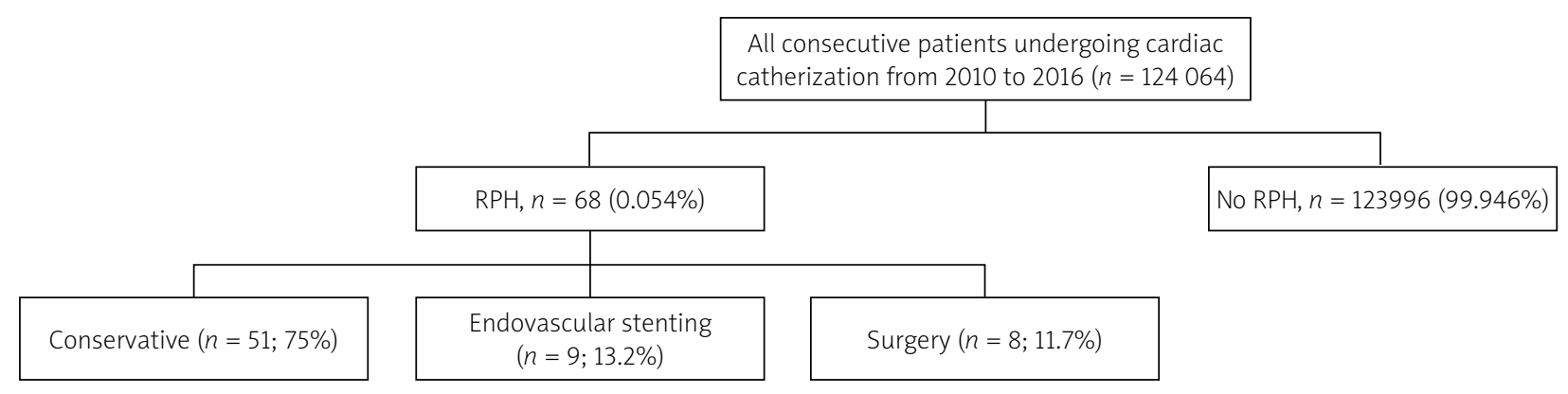

Figure 1. Choice of patient cohort and initial management of RPH cases 
RPH had a mean age of $59 \pm 6.7$, while there was no statistically significant difference between them and those with RPH $(p=0.526)$. Along the same lines, $68.9 \%$ of the patients with no RPH development were male, while $31.3 \%$ were female; there was no statistically significant difference between them and the RPH group ( $p=0.122$ ). During the study, VCDs were used in 1325 patients in our hospital. Only 1 patient with VCD developed RPH. There was no significant difference in the use of VCD between these two groups ( $p=0.745)$

There were $51 \mathrm{RPH}$ patients in group 1 who had been treated conservatively, 9 patients in group 2 who had received angiographic stents, and 8 patients in group 3 who had undergone surgical treatment.

\section{Baseline demographics and characteristics}

Of the $68 \mathrm{RPH}$ patients (mean age of $63.13 \pm 13.89$ ), $41(60.3 \%)$ were male and 27 (39.7\%) were female. Indications for CC were myocardial infarction in 24 (35.3\%) patients, acute coronary syndrome in 18 (26.5\%) patients, stable angina pectoris in $15(22.1 \%)$ patients, hemodynamic studies for valvular heart disease in 8 (11.8\%) patients, and right cardiac pathology in 3 (4.4\%) patients. Forty-six of these patients had received percu- taneous coronary procedures; however, there were no statistically significant differences among the groups $(p=0.341)$. In addition, there were no statistically significant differences between the groups' clinical and demographic characteristics (Table I).

The most common symptom in the patients was abdominal pain and the most common physical sign was abdominal tenderness. Symptoms and findings of RPH cases are summarized in Table II.

\section{In-hospital outcomes}

Pre-procedure (at the time of RPH diagnosis) and follow-up laboratory results of the patients revealed that the only statistically significant difference between the groups was that seen in the highest cre $(\mathrm{mg} / \mathrm{dl})$ value $(p=0.013)$. An examination of abdominal CT results showing the maximum hematoma sizes in patients of all the groups revealed that there were no statistically significant differences between the groups, although the sizes of the hematomas in group 3 were larger than those of the other groups $(p=0.326)$. Moreover, despite the fact that $21(30.9 \%)$ patients were diagnosed within the first $24 \mathrm{~h}$ following the procedure, there were no significant differences among the groups $(p=0.143)$.

Table I. Clinical and demographic characteristics of RPH patients according to treatment methods

\begin{tabular}{|c|c|c|c|c|c|}
\hline Variable & & Conservative $(n=51)$ & Stenting $(n=9)$ & Surgery $(n=8)$ & $P$-value \\
\hline \multirow[t]{2}{*}{ Gender } & Male & $29(56.9 \%)$ & $6(66.7 \%)$ & $6(75 \%)$ & \multirow[t]{2}{*}{0.569} \\
\hline & Female & $22(43.1 \%)$ & $3(33.3 \%)$ & $2(25 \%)$ & \\
\hline Age & & $64.4 \pm 13.04$ & $62.1 \pm 16.3$ & $55.7 \pm 15.8$ & 0.252 \\
\hline \multirow[t]{6}{*}{ Comorbidities } & $\mathrm{DM}$ & $18(35.3 \%)$ & $3(33.3 \%)$ & $2(25 \%)$ & 0.849 \\
\hline & HT & $27(52.9 \%)$ & $4(44.4 \%)$ & $2(25 \%)$ & 0.328 \\
\hline & COPD & $8(15.7 \%)$ & 0 & $3(37.5 \%)$ & 0.109 \\
\hline & CRF & $5(9.8 \%)$ & $1(11.1 \%)$ & $1(12.5 \%)$ & 0.970 \\
\hline & CVD & $5(9.8 \%)$ & 0 & $1(12.5 \%)$ & 0.587 \\
\hline & $A F$ & $2(3.9 \%)$ & $2(22.2 \%)$ & $1(12.5 \%)$ & 0.128 \\
\hline Weight [kg] & & $80.63 \pm 10.94$ & $74.44 \pm 9.4$ & $78.13 \pm 10.96$ & 0.270 \\
\hline BMI $\left[\mathrm{kg} / \mathrm{m}^{2}\right]$ & & $27.9 \pm 4.39$ & $25.7 \pm 4.2$ & $26.49 \pm 4.04$ & 0.297 \\
\hline LVEF & & $55.2 \pm 6.8$ & $53.89 \pm 10.8$ & $53.75 \pm 13.02$ & 0.840 \\
\hline \multirow[t]{5}{*}{ Indication for $\mathrm{PCl}$} & Acute MI & $20(39.2 \%)$ & $1(11.1 \%)$ & $3(37.5 \%)$ & \multirow[t]{5}{*}{0.716} \\
\hline & Unstable angina & $13(25.5 \%)$ & $3(33.3 \%)$ & $2(25 \%)$ & \\
\hline & Stable angina & $11(21.61 \%)$ & $3(33.3 \%)$ & $1(12.5 \%)$ & \\
\hline & Valvular heart disease & $5(9.8 \%)$ & $2(22.2 \%)$ & $1(12.5 \%)$ & \\
\hline & Right heart catheterization & $2(3.9 \%)$ & 0 & $1(12.5 \%)$ & \\
\hline $\mathrm{PCl}$ & & $33(64.7 \%)$ & $8(88.9 \%)$ & $5(62.5 \%)$ & 0.341 \\
\hline
\end{tabular}

$D M$ - diabetes mellitus, HT - hypertension, COPD - chronic obstructive pulmonary disease, CRF - chronic renal failure, CVD - cerebrovascular disease, AF - atrial fibrillation, $B M I$ - body mass index, $L V E F$ - left ventricle ejection fraction, $M I$ - myocardial infarction, $P C l$ - percutaneous coronary intervention. 
The RBCPs (unit), duration of hospitalization, and rate of mortality were statistically significantly higher in group 3 compared to the other groups. In-hospital results are summarized in Table III.

Five of the 8 patients who had received surgical treatment underwent the operations because of abdominal compartment syndrome due to giant retroperitoneal hematomas (an increase in intra-abdominal pressure and accompanying organ perfusion disorder), two underwent surgery because of instability following failed endovascular treatment, and one patient underwent surgery because of indications of nerve compression.

The results of the significance tests, which were conducted for the coefficients of the variables covered by each univariate model (Table IV), revealed that age $\geq 65$, chronic obstructive pulmonary disease (COPD), a mean hematocrit drop $\geq 15 \%$, a post-diagnostic highest creatinine $\geq 2 \mathrm{mg} / \mathrm{dl}$, RBCP units $\geq 10$, a diagnosis more than $24 \mathrm{~h}$ following CC, and surgical treatment were found to be in a statistically significant relationship with the dependent variable. These variables were set as candidates for the multivariate model.

The results of the multivariate analysis of the RPH dependent mortality-related factors, on the other hand, through logistic regression analysis (Table $V$ ), revealed post-procedural highest creatinine $\mathrm{mg} / \mathrm{dl} \geq 2$ and RBCPs $\geq 10$ as independent risk factors. Other variables ascertained to be significant via the univariate analysis, however, were not found to be significant based on the multivariate analysis.
Table II. Symptoms and physical findings of patients with RPH

\begin{tabular}{llc} 
Clinical features & Cases, $\boldsymbol{n}$ (\%) \\
\hline Symptoms & Abdominal pain & $35(51.4)$ \\
\cline { 2 - 3 } & Groin pain & $15(22)$ \\
\cline { 2 - 3 } & Back pain & $12(17.6)$ \\
\cline { 2 - 3 } & Nausea/vomiting & $10(14.7)$ \\
\cline { 2 - 3 } & Lower extremity pain & $8(11.7)$ \\
\hline Shysical & Hypotension & $50(73.5)$ \\
\cline { 2 - 3 } & Abdominal tenderness & $38(55.8)$ \\
\cline { 2 - 3 } & Groin hematoma & $17(25)$ \\
\cline { 2 - 3 } & Flank ecchymosis & $1(14.7)$ \\
\cline { 2 - 3 } & Femoral nerve palsy & $1(1.47)$
\end{tabular}

The classification table, which was obtained by the inclusion of the variables covered by the mortality development model in the model, is shown in Table VI. The rate of correct classification was found to be $88.2 \%$. Results of the Hosmer-Lemeshow test, which was used to assess the goodness of fit of the model, showed that the model was adequate $\left(\chi^{2}=2.839, \mathrm{~d} f=7\right.$, and $\left.p=0.899\right)$.

\section{Discussion}

Non-cardiac complications mostly were related to femoral artery puncture site in CCs by which the femoral

Table III. Hospital outcomes according to groups

\begin{tabular}{|c|c|c|c|c|c|}
\hline \multicolumn{2}{|l|}{ Variable } & \multirow{2}{*}{$\frac{\text { Conservative }(n=51)}{37.6 \pm 3.9}$} & \multirow{2}{*}{$\frac{\text { Stenting }(n=9)}{37.6 \pm 5.5}$} & \multirow{2}{*}{$\frac{\text { Surgery }(n=8)}{38.6 \pm 7.5}$} & \multirow{2}{*}{$\frac{P \text {-value }}{0.852}$} \\
\hline Laboratory & Baseline hematocrit (\%) & & & & \\
\hline & Baseline platelet count [G/I] & $244.8 \pm 71$ & $207.8 \pm 49.3$ & $238.5 \pm 116.8$ & 0.341 \\
\hline & +Baseline creatinine [mg/dl] & $0.83 \pm 0.22$ & $1.01 \pm 0.26$ & $0.92 \pm 0.20$ & 0.117 \\
\hline & Nadir hematocrit (\%) & $23.4 \pm 4.6$ & $21.3 \pm 3.8$ & $20.9 \pm 5.8$ & 0.250 \\
\hline & Nadir platelet count [G/l] & $141.9 \pm 82.2$ & $105.3 \pm 56.3$ & $104.2 \pm 80.5$ & 0.202 \\
\hline & Mean hematocrit drop (\%) & $14.07 \pm 5.2$ & $16.3 \pm 4.5$ & $17.6 \pm 10.3$ & 0.506 \\
\hline & ${ }^{+}$Highest creatinine $[\mathrm{mg} / \mathrm{dl}]$ & $1.56 \pm 1.31$ & $1.46 \pm 0.46$ & $3.1 \pm 1.55$ & $0.013^{*}$ \\
\hline & Leukocytes at diagnosis & $18780 \pm 32356$ & $17500 \pm 8035$ & $20150 \pm 9342$ & 0.982 \\
\hline \multicolumn{2}{|c|}{ Maximum size detected in abdominal CT [mm] } & $142 \pm 59$ & $138 \pm 42.8$ & $189.7 \pm 83.5$ & 0.326 \\
\hline \multicolumn{2}{|c|}{ Number of RBCP transfused [U] } & $5 \pm 5.7$ & $5.7 \pm 4.1$ & $10.1 \pm 8.06$ & $0.043^{*}$ \\
\hline \multicolumn{2}{|c|}{ Time from procedure to $\mathrm{CT}$, first $24 \mathrm{~h}$} & 19 (37.3\%) & 1 (11.1\%) & $1(12.5 \%)$ & 0.143 \\
\hline \multicolumn{2}{|c|}{ Length of stay in hospital, mean \pm SD [days] } & $18 \pm 13$ & $14 \pm 8$ & $51 \pm 47$ & $0.007^{*}$ \\
\hline \multicolumn{2}{|l|}{ Mortality } & 7 (13.7\%) & $2(22.2 \%)$ & $5(62.5 \%)$ & $0.006^{\star}$ \\
\hline
\end{tabular}

$C T$ - computed tomography, RBCP-red blood cell packs, ${ }^{+}$Patients with CRF were not included in the creatinine-related evaluation. *Differences between the groups statistically significant $p<0.05$. 
Table IV. Univariate logistic regression model analysis of mortality related factors in patients with RPH

\begin{tabular}{|c|c|c|c|c|c|c|c|}
\hline \multicolumn{2}{|l|}{ Variable } & Total & No mortality & Mortality & Odds ratio & $95 \% \mathrm{Cl}$ & $P$-value \\
\hline \multicolumn{2}{|l|}{ Age ( $\geq 65$ years) } & 36 & $25(69.44 \%)$ & $11(30.55 \%)$ & 4.255 & $0.059-0.938$ & $0.04^{*}$ \\
\hline \multicolumn{2}{|l|}{ Male sex } & 41 & 33 (80.5\%) & $8(19.5 \%)$ & 0.848 & $0.258-2.793$ & 0.787 \\
\hline \multicolumn{2}{|l|}{ LVEF $<55$} & 23 & $17(31.5 \%)$ & $6(42.9 \%)$ & 1.632 & $0.490-5.443$ & 0.425 \\
\hline \multicolumn{2}{|l|}{$\mathrm{BMI}(\geq 25 \mathrm{~kg} / \mathrm{m} 2)$} & 46 & $36(78.2 \%)$ & $10(21.7 \%)$ & 0.800 & $0.220-2.907$ & 0.735 \\
\hline \multicolumn{2}{|l|}{ HT } & 33 & $26(48.1 \%)$ & $7(50 \%)$ & 0.929 & $0.287-3.009$ & 0.902 \\
\hline \multicolumn{2}{|l|}{ DM } & 23 & $19(35.2 \%)$ & $4(28.6 \%)$ & 1.357 & $0.375-4.916$ & 0.642 \\
\hline \multicolumn{2}{|l|}{ CRF } & 7 & $5(71.4 \%)$ & $2(28.6 \%)$ & 0.612 & $0.106-3.548$ & 0.584 \\
\hline \multicolumn{2}{|l|}{$A F$} & 5 & $3(5.6 \%)$ & $2(14.3 \%)$ & 0.353 & $0.053-2.351$ & 0.282 \\
\hline \multicolumn{2}{|l|}{ COPD } & 11 & $5(9.3 \%)$ & $6(42.9 \%)$ & 7.352 & $0.033-0.553$ & $0.005^{*}$ \\
\hline \multicolumn{2}{|l|}{ CVD } & 6 & 5 (9.3\%) & $1(7.1 \%)$ & 1.327 & $0.142-12.366$ & 0.804 \\
\hline \multicolumn{2}{|c|}{ Localization of hematoma (right) } & 37 & $28(52.8 \%)$ & $9(64.3 \%)$ & 0.622 & $0.184-2.105$ & 0.446 \\
\hline \multicolumn{2}{|c|}{ Mean hematocrit drop $\geq 15 \%$} & 34 & $24(70.5 \%)$ & $10(29.4 \%)$ & 0.320 & $0.089-1.148$ & $0.080^{*}$ \\
\hline \multicolumn{2}{|c|}{$\begin{array}{l}\text { Highest creatinine } \geq 2 \mathrm{mg} / \mathrm{dl} \\
\text { (CRF patients were not included) }\end{array}$} & 23 & $11(20.4 \%)$ & $12(85.7 \%)$ & 23.255 & $0.008-0.219$ & $0.001^{*}$ \\
\hline \multicolumn{2}{|c|}{ RBC transfusion $\geq 10 \mathrm{U}$} & 9 & $2(3.7 \%)$ & $7(50 \%)$ & 26.315 & $0.007-0.223$ & $0.001^{*}$ \\
\hline \multicolumn{2}{|c|}{ RPH diagnosis more than $24 \mathrm{~h}$ following CC } & 47 & $34(72.3 \%)$ & $13(27.7 \%)$ & 0.131 & $0.016-1.076$ & $0.059^{*}$ \\
\hline \multirow{5}{*}{$\begin{array}{l}\text { Pre-procedure } \\
\text { medical treatment }\end{array}$} & IV heparin & 7 & $5(71.4 \%)$ & $2(28.6 \%)$ & 0.612 & $0.106-3.548$ & 0.584 \\
\hline & Clopidogrel & 41 & $33(61.1 \%)$ & $7(50 \%)$ & 1.571 & $0.482-5.124$ & 0.454 \\
\hline & LMW heparin & 29 & $23(42.6 \%)$ & $6(42.9 \%)$ & 0.989 & $0.302-3.245$ & 0.986 \\
\hline & Warfarin & 6 & $6(11.1 \%)$ & 0 & 1.625 & $0.179-14.723$ & 0.666 \\
\hline & ASA & 42 & $36(66.7 \%)$ & $6(42.9 \%)$ & 1.842 & $0.562-6.038$ & 0.313 \\
\hline \multicolumn{2}{|l|}{$\mathrm{PCl}$} & 46 & 38 (70.4\%) & $8(57.1 \%)$ & 1.781 & $0.532-5.967$ & 0.349 \\
\hline \multicolumn{2}{|l|}{ Surgical treatment } & 8 & $3(37.5 \%)$ & $5(62.5)$ & 9.433 & $0.021-0.523$ & $0.006^{*}$ \\
\hline
\end{tabular}

LVEF-left ventricle ejection fraction, BMI - body mass index. HT - hypertension, DM - diabetes mellitus, CRF - chronic renal failure, AF - atrial fibrillation, COPD - chronic obstructive pulmonary disease, CVD - cerebrovascular disease, CC - cardiac catheterization, $P C I$ - percutaneous coronary intervention, IV - intravenous, LMW - low molecular weight, ASA - acetylsalicylic acid. *Differences between the groups statistically significant $p<0.05$.

Table V. Multiple logistic regression model analysis of mortality related factors in patients with $\mathrm{RPH}$

\begin{tabular}{lccc} 
Variable & OR & 95\% Cl & P-value \\
\hline Age ( $\geq 65$ years) & 0.113 & $0.007-1.927$ & 0.132 \\
\hline COPD & 0.172 & $0.004-8.014$ & 0.369 \\
\hline Mean hematocrit drop $\geq 15 \%$ & 0.176 & $0.017-1.804$ & 0.144 \\
\hline Highest creatinine $\geq 2 \mathrm{mg} / \mathrm{dl}$ & 25.64 & $0.003-0.586$ & 0.019 \\
\hline RBC transfusion $\geq 10 \mathrm{U}$ & 41.66 & $0.001-0.692$ & 0.030 \\
\hline $\begin{array}{l}\text { Diagnosis outside the first } \\
\text { 24 h after PCl }\end{array}$ & 0.265 & $0.019-3.759$ & 0.327 \\
\hline $\begin{array}{l}\text { Surgical treatment } \\
\text { Sult }\end{array}$ & 0.527 & $0.012-21.699$ & 0.736
\end{tabular}

COPD - chronic obstructive pulmonary disease, $P C I$ - percutaneous coronary intervention, $R B C$ - red blood cell.
Table VI. Correct classification ratio table of mortality model

\begin{tabular}{lccc}
\hline \multirow{2}{*}{ Observed mortality } & \multicolumn{3}{c}{ Predicted mortality } \\
\cline { 2 - 4 } & None & Present & Verification ratio \\
\hline None & 51 & 3 & $94.4 \%$ \\
\hline Present & 5 & 9 & $64.3 \%$ \\
\hline $\begin{array}{l}\text { Overall classification } \\
\text { ratio }\end{array}$ & & & $88.2 \%$
\end{tabular}

artery route was used. Although its incidence generally varies according to study populations, the rate has been reported to be between $0 \%$ and $17 \%$ [6]. These vascular complications can be classified as minor or major. Minor complications include minor hemorrhaging, ecchymosis, and stable hematoma, while pseudoaneurysm, arteriove- 
nous (AV) fistula, hematoma-necessitating transfusion, retroperitoneal hemorrhaging, arterial dissection, emboli, thrombosis, infection, and extremity ischemia can be listed among the major complications. Hemorrhaging, the most common extra-cardiac complication observed in catheterizations using a transfemoral approach, can appear as a stable or unstable hematoma, uncontrolled hemorrhaging, pseudoaneurysm, or retroperitoneal hemorrhaging [7, 8]. Major bleeding complications are also less common in procedures using the radial artery [9].

The risk factors for vascular complications of transfemoral catheterization can be divided into two types: patient-related and procedure-related. Patient-related risk factors include female gender, low body weight, obesity, low body surface area, advanced age, peripheral vascular disease, renal failure or increased cre, and low plt count [10]. Procedure-dependent factors include high-dose and long-term anticoagulant administration, thrombolytic agent administration, glycoprotein (Gp) Ilb/Illa inhibitors (especially abciximab), wide arterial sheath, simultaneous multiple invasive interventions, catheter sheath left in the groin for an extended period of time, prolonged procedure duration, recurrent $\mathrm{PCl}$, and location of the arterial puncture [10,11].

The RPH is most commonly observed as a complication of femoral arterial catheterization and pelvic or lumbar trauma. Spontaneous hemorrhaging without a marked determinant factor is called spontaneous $R P H$, a condition most frequently related to anticoagulant or antiaggregant treatment, hemodialysis, and bleeding diathesis [12-14].

Consistent with previous literature, we believe that $\mathrm{RPH}$ occurring in patients after CC is a rare complication of the procedure [1-4]. Although RPH can accompany subclinical bleeding symptoms, such as pain in the lower abdominal quadrant, femoral neuropathy, hypotension, nausea, and inguinal sensitivity or fullness [1, 15], our study revealed that there is a significant relationship between RPH and the risk of mortality.

We ascertained that the incidence of RPH at our center during the 6 years covered by the study was $0.054 \%$ (68 RPH cases out of a total of 124,064 CC cases). Previous studies have reported varying incidence rates for CC-related RPH. Sreeram et al. [15] reported a CC-related $\mathrm{RPH}$ incidence rate of $0.15 \%$, while Kent et al. [1] reported a rate of $0.47 \%$, Frank et al. [16] reported a rate of $0.13 \%$, and Eisen et al. [2] reported a rate of $0.18 \%$. Other studies have also reported a higher rate of $\mathrm{RPH}$ incidence after $\mathrm{PCl}[3,5]$.

Although the controversy surrounding the treatment of $\mathrm{RPH}$ continues, conservative treatment is generally recommended for hemodynamically stable patients with no active bleeding symptoms $[1,2,5,16,17]$. There are no specific guidelines as to which patients should receive endovascular or open surgery, or under which cir- cumstances it should be performed; in 1993, however, it was reported that endovascular treatment is an effective method for both iatrogenic and spontaneous RPH cases [17-22]. In their 2016 study, Seropian et al. [22] recommended that patients suspected of having RPH after PCI, and whose hemodynamic stability could not be achieved within the first $30 \mathrm{~min}$, should be transferred to the catheterization laboratory for endovascular treatment without further tests (USG, CT). Surgical treatment, on the other hand, has been recommended for hemodynamically unstable patients with failed endovascular treatment and for patients with abdominal compartment syndrome $[2,17,22]$.

Seventy-five percent of the patients with RPH had received conservative treatment, $13.2 \%$ had received angiographic stents, and $11.7 \%$ had undergone surgical treatment. A higher rate of in-hospital mortality inclination was observed in RPH patients treated surgically compared with those who had received medical and stent treatments. It is probable that accompanying cardiac problems, massive transfusion, and renal function disorders in these patients, for whom hemodynamic stability could not be achieved, increased the rate of mortality. We believe, however, that surgical treatment should be conducted when necessary (e.g., for unstable patients after endovascular stenting and for patients with abdominal compartment syndrome).

There were some significant limitations of our study. Ours was a retrospective observational study, which implies limitations in the study design. The main limitation was the absence of a control group paired with patients without bleeding. Furthermore, the population of our study was heterogeneous, as it included CC patients with various etiologies, including right cardiac catheterization. An additional limitation was the possibility that some patients with bleeding but not diagnosed might have gone unnoticed. These patients could include those with small-scale hemorrhaging, normal clinical results, and an absence of radiological screening assessments. Finally, we did not have specific data on the location of femoral arterial puncture in patients with $\mathrm{RPH}$.

\section{Conclusions}

There is no randomized study that can guide the treatment strategies for RPH: evidence is based on small case series or on isolated case reports. Conservative treatment methods, such as follow-up in the intensive care unit, fluid resuscitation, blood transfusion, and the reversal of anticoagulant and/or antiaggregant treatment, have been used as effective strategies in most patients. In the cases of patients whose hemodynamic stability cannot be achieved in spite of aggressive resuscitation, however, we believe that the next step should be a percutaneous approach and that open surgery should be performed 
only on patients whose bleeding cannot be controlled or who have abdominal compartment syndrome.

\section{Conflict of interest}

The authors declare no conflict of interest.

\section{References}

1. Kent KC, Moscucci M, Mansour KA, et al. Retroperitoneal hematoma after cardiac catheterization: prevalence, risk factors, and optimal management. J Vasc Surg 1994; 20: 905-10.

2. Eisen A, Kornowski R, Vaduganathan $M$, et al. Retroperitoneal bleeding after cardiac catheterization: a 7-year descriptive single-center experience. Cardiology 2013; 125: 217-22.

3. Tiroch KA, Arora N, Matheny ME, et al. Risk predictors of retroperitoneal hemorrhage following percutaneous coronary intervention. Am J Cardiol 2008; 102: 1473-6.

4. Trimarchi S, Smith DE, Shar D, et al. Retroperitoneal hematoma after percutaneous coronary intervention: prevalence, risk factors, management, outcomes, and predictors of mortality. JACC Cardiovasc Interv 2010; 3: 845-50.

5. Farouque HM, Tremmel JA, RaissiShabari F, et al. Risk factors for the development of retroperitoneal hematoma after percutaneous coronary intervention in the era of glycoprotein $\mathrm{Ilb} / \mathrm{llla}$ inhibitors and vascular closure devices. J Am Coll Cardiol 2005; 45: 363-8.

6. Sherev DA, Shaw RE, Brent BN. Angiographic predictors of femoral access site complications: implication for planned percutaneous coronary intervention. Catheter Cardiovasc Interv 2005; 65: 196-202.

7. Samal AK, White CJ. Percutaneous management of access site complications. Catheter Cardiovasc Interv 2002; 57: 12-23.

8. Bhatty S, Cooke R, Shetty R, et al. Femoral vascular access-site complications in the cardiac catheterization laboratory: diagnosis and management. Interv Cardiol 2011; 3: 503-14.

9. Andó G, Costa F. Bleeding risk stratification in acute coronary syndromes. Is it still valid in the era of the radial approach? Postep Kardiol Interw 2015; 11: 170-3.

10. Piper WD, Malenka DJ, Ryan TJ, et al. Predicting vascular complications in percutaneous coronary interventions. Am Heart J 2003; 145: 1022-9.

11. Wiley JM, White CJ, Uretsky BF. Non coronary complications of coronary intervention. Catheter Cardiovasc Interv 2002; 57: 257-65.

12. Jurisic D, Doko M, Glavan E, et al. Spontaneous retroperitoneal haematoma associated with clopidogrel therapy mimicking acute appendicitis. Br J Clin Pharmacol 2006; 62: 248-9.

13. Bhasin HK, Dana CL. Spontaneous retroperitoneal hemorrhage in chronically hemodialyzed patients. Nephron 1978; 22: 322-7.

14. Ivascu FA, Janczyk RJ, Bair HA, et al. Spontaneous retroperitoneal hemorrhage. Am J Surg 2005; 189: 345-7.

15. Sreeram S, Lumsden AB, Miller JS, et al. Retroperitoneal hematoma following femoral arterial catheterization: a serious and often fatal complication. Am Surg 1993; 59: 94-8.

16. Frank JJ, Kamalakannan D, Kodenchery M, et al. Retroperitoneal hematoma in patients undergoing cardiac catheterization. J Interv Cardiol 2010; 23: 569-74.

17. Ellis SG, Bhatt D, Kapadia S, et al. Correlates and outcomes of retroperitoneal hemorrhage complicating percutaneous coronary intervention. Catheter Cardiovasc Interv 2006; 67: 541-5.
18. Mak GY, Daly B, Chan W, et al. Percutaneous treatment of post catheterization massive retroperitoneal hemorrhage. Cathet Cardiovasc Diagn 1993; 29: 40-3.

19. Silva JA, Stant J, Ramee SR. Endovascular treatment of a massive retroperitoneal bleeding: successful balloon-catheter delivery of intra-arterial thrombin. Catheter Cardiovasc Interv 2005; 64: 218-22.

20. Samal AK, White CJ. Percutaneous management of access site complications. Catheter Cardiovasc Interv 2002; 57: 12-23.

21. Pathi R, Voyvodic F, Thompson WR. Spontaneous extraperitoneal haemorrhage: computed tomography diagnosis and treatment by selective arterial embolization. Australas Radiol 2004; 48: 123-8.

22. Seropian IM, Angiolillo DJ, Zenni MM, et al. Should endovascular approach be the first line of treatment for retroperitoneal bleeding with hemodynamic shock following percutaneous intervention? A case series. Catheter Cardiovasc Interv 2016; 90: 104-11. 